\section{A Rare Differential for Metastatic Melanoma}

This case describes a 50 year old man with no previous history of melanoma or non-melanoma skin cancer, who was referred with a suspected diagnosis of metastatic amelanotic melanoma with an unknown primary. Prior to referral he had no skin biopsy, no pathologic diagnosis of primary or metastatic melanoma and an FNA inconclusive for melanoma. He had a 10 week history of an ulcerated lesion on his forearm with ipsilateral epitrochlear and axillary lymph nodes suspicious both clinically and on CT scan. He was referred from a major University hospital after having been seen by both a Plastic Surgeon and Dermatologist. This case is important as this diagnosis was assumed in the absence of definitive tissue diagnosis due to an inconclusive FNA. The patient was significantly distressed with the assumed diagnosis of melanoma with nodal metastases implicating the need for significant surgery as well as a significantly reduced life expectancy.

Epitrochlear lymphadenopathy in distal upper limb metastatic melanoma is considered rare and the literature quotes variable rates of between $3 \%$ and $20 \%$ [1-3]. Axillary lymphadenopathy is a more common finding. Following referral, a detailed history was taken and significantly enlarged lymph nodes were found to be mildly tender on palpation; $5 \mathrm{~cm}^{2}$ at the elbow and $3 \mathrm{~cm}^{2}$ in the axilla. His history revealed that he was otherwise fit and well with no previous history of skin oncology, trauma, insect bites or animal scratches. He did report keeping pet cats and rabbits. An excision biopsy of the forearm lesion was performed under local anaesthetic and specimens were sent for both histopathology and microbiological analysis. Repeat US guided FNAC of the epitrochlear lymph nodes was undertaken. He underwent routine haematological and biochemical blood tests as well as microbial serology for Bartonella henselae. He was commenced on an oral course of Azithromycin. Histopathological analysis of the lesion revealed proliferation of fibroblasts/myofibroblasts and vessels with noncaseating granulomata. Special stains showed no obvious fungal organisms. US guided FNAC demonstrated relatively normal ultrasound morphology of the axillary lymphnodes, with uniform low echogenicity, and were not typical of metastases. Cytology showed epithelioid histiocytes forming granulomata. No malignant cells were identified. Appearances were consistent with granulomatous lymphadenitis.

Routine blood tests were normal, however, Bartonella serology returned with the parameters as shown in Table 1 . Levels were suggestive of Bartonella infection of an indeterminate timeframe.

Table 1: Bartonella serology.

\begin{tabular}{|c|c|}
\hline Organism & Titre \\
\hline Bartonella henselae IgM & $<20$ \\
\hline Bartonella henselae IgG & $128^{*}$ \\
\hline Bartonella quintana IgM & $<20$ \\
\hline Bartonella quintana IgG & $<64$ \\
\hline
\end{tabular}

\section{Journal of}

Clinical \& Investigative Dermatology

\author{
Ingrid Kieran*, Patrick Shenjere and Damir Kosutic \\ Christie Hospital, Wilmslow Road, Manchester, UK
}

*Address for Correspondence

Ingrid Kieran, Christie Hospital, Wilmslow Road, Manchester M20 4BX, UK, Tel: +07809509517; E-mail: ingridkieran@nhs.net

Submission: 25 January, 2017

Accepted: 08 March, 2017

Published: 17 March, 2017

Copyright: ๑ 2017 Kieran I, et al. This is an open access article distributed under the Creative Commons Attribution License, which permits unrestricted use, distribution, and reproduction in any medium, provided the original work is properly cited.

The initial diagnosis for this lesion was presumed to be an aggressive skin oncology. While urgency in diagnosing suspect skin cancers is important, this case highlights the importance of nonmalignant causes of epitrochlear and axillary lymphadenopathy such as cat scratch disease. This differential diagnosis should be considered by surgeons and dermatologists prior to establishing the diagnosis of metastatic malignancy in the setting of a tertiary cancer centre. Furthermore, a detailed history including social circumstances may have raised suspicion for Bartonella infection prior to embarking on sophisticated imaging. An expedient excision biopsy should be performed for definitive histology if considering melanoma diagnosis prior to widespread imaging as in this case this caused the patient significant psychological distress at the possibility of metastatic disease. This case also suggests potential differences in the experience of different radiological centers and reporting of metastatic appearances of lymphnodes on both US and CT modalities.

In conclusion, this was an unusual presentation of cat scratch disease, however, a smooth, tender, enlarged epitrochlear lymph node should raise suspicion of both malignant and nonmalignant causes for lymphadenopathy, including cat scratch disease [4]. Melanoma surgeons should consider this in the differential diagnosis to aid investigation. Also, while referral of suspected metastatic melanoma should not be delayed to the appropriate centre for further management, diagnosis should be reserved until definitive tissue is available. This is in accordance with NICE guidelines for melanoma assessment which state that diagnosis should be based on histology, clinical features and behavior [5].

\section{References}

1. Moloney DM, Overstall S, Allan R, Powell BW (2001) An aberrant lymph node containing metastatic melanoma detected by sentinel node biopsy. $\mathrm{Br} \mathrm{J}$ Plast Surg 54: 638-640.

2. Hunt JA, Thompson JF, Uren RF, Howman-Giles R, Harman CR (1998) Epitrochlear lymph nodes as a site of melanoma metastasis. Ann Surg Oncol 5: 248-252.

3. Tanabe KK (1997) Lymphatic mapping and epitrochlear lymph node dissection for melanoma. Surgery 121: 102-104.

4. Graveleau J, Grossi O, Lefebvre M, Redon H, Caignon JM, et al. (2011) 
Citation: Kieran I, Shenjere P, Kosutic D. A Rare Differential for Metastatic Melanoma. J Clin Investigat Dermatol. $2017 ;$ 5(1): 2.

ISSN: 2373-1044

Vertebral osteomyelitis: an unusual presentation of Bartonella henselae infection. Semin Arthritis Rheum 41: 511-516.
5. NICE guideline (2015) Melanoma: assessment and management [NG14] Assessing and managing atypical spitzoid lesions. Section 1.2.5. 